\title{
Trial discontinuation: lessons for future trial design?
}

Clin. Invest. (2012) 2(2), 147-152

Background: The current therapeutic arsenal still does not fulfill the therapeutic needs of inflammatory bowel disease patients. Although new drugs are constantly being developed, many will never reach the market. In this review we will search for reasons for discontinuing promising clinical trials and offer recommendation for future trials. Methods: The website clinicaltrials.gov was searched for interventional trials on novel inflammatory bowel disease therapies. Included were discontinued 'Crohn's disease' and/or 'colitis, ulcerative' trials, started between July 1996 and October 2011 and discontinued. Pubmed was searched for publications to elucidate reasons for discontinuation. Results: One hundred and ninety one novel drug trials were published on clinicaltrials. gov, of which $24(12.6 \%)$ were interrupted. The most common reason for discontinuation was lack of efficacy. Conclusion: Translation from bench to bedside is not always feasible, animal models come with restrictions. For better treatments, personalized medicine will be the future.

Keywords: clinical trials • Crohn's disease • inflammatory bowel disease - translational research • trial design • ulcerative colitis

Inflammatory bowel disease (IBD) is a chronic disease of the gastrointestinal tract and comprises of Crohn's disease (CD), ulcerative colitis (UC) and unclassified colitis. IBD patients experience periods of remission of their disease but are also prone to relapse and it was demonstrated that IBD can evolve to a more complicated phenotype [1-4]. The highest incidence rates are reported from Northern Europe, the UK and North America, ranging from 6.6 to 15.6 cases per 100,000 person years [5]. The impact on physical, social, as well as the emotional well-being of patients is substantial and the disease profoundly decreases the quality of life [6].

At present, no therapeutic intervention exists that can lead to a definitive cure. As such, the current treatment goal is to provide long-term remission. Towards this aim, the armamentarium has been expanded with novel and more potent drugs. Although many drugs have been used in the treatment of IBD, none has, so far, been shown to modify the natural history of the disease or to maintain a stable remission over time [7]. Population-based studies show that, using the conventional therapies, only $42 \%$ of the patients with $\mathrm{CD}$ are symptom free 2 years after initial diagnosis and only $12 \%$ after 10 years. Besides this, $10 \%$ of patients have continuously active disease at 2 years after initial diagnosis and $1 \%$ at 10 years [8]. Since steroids cannot be used as maintenance therapy for remission, immunosuppression with thiopurinic agents, such as azathioprine, mercaptopurine or methotrexate have been widely used, even though all have limited value for induction of remission and can only benefit less than half of the patients who suffer from steroid dependency or resistance [9]. Resection of the inflamed bowel does not interrupt the progression of the disease in CD patients [8]. Cosnes et al. investigated the need for intestinal surgery from 1987 up to 2002 [10]. Although the 5-year cumulative probability of the immunosuppressant use increased from 0.13 to 0.56 , the cumulative risk of intestinal surgery remained unchanged over
Veerle JAA Nuij, Colin J de Haar \& C Janneke van der Woude*

Department of Gastroenterology \& Hepatology, Erasmus Medical Center, 3015 CE Rotterdam, The Netherlands

*Author for correspondence:

E-mail:v.nuij@erasmusmc.nl

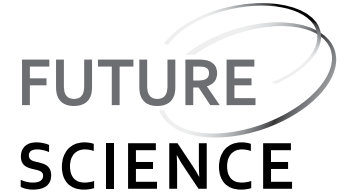

part of fsg 
time [10].

Anti-TNF- $\alpha$ therapy proved to be a potent treatment for IBD; however, in $30 \%$ of patients it is not effective and a substantial proportion of patients may experience loss of response and/or side-effects [11-14], which often make surgery unavoidable. As such, expanding our therapeutic arsenal is still necessary.

It takes many years for potential drugs to enter human phase clinical trials. Even after reaching this phase, many drugs will never reach the market. Currently, discussion arises on the optimal trial design in IBD. In this review we will try to elucidate reasons for discontinuing trials and based on this knowledge will give some recommendations for designing future trials in IBD patients.

\section{Materials \& methods}

The Clinicaltrials.gov website [101] was searched for trials listed under the conditions 'Crohn's disease' and/or 'colitis, ulcerative' between July 1996 and October 2011. Only interventional studies on new (unregistered) treatments were included. Nondrug trials (dietary supplements, probiotics, devices and stem cells) were excluded. Also excluded were trials where there was no follow-up reported within the last 2 years.

According to the trial Phase (I, II, III) the trials were further divided into: completed, ongoing, discontinued and unknown. Only discontinued trials were further studied. The Pubmed database was used

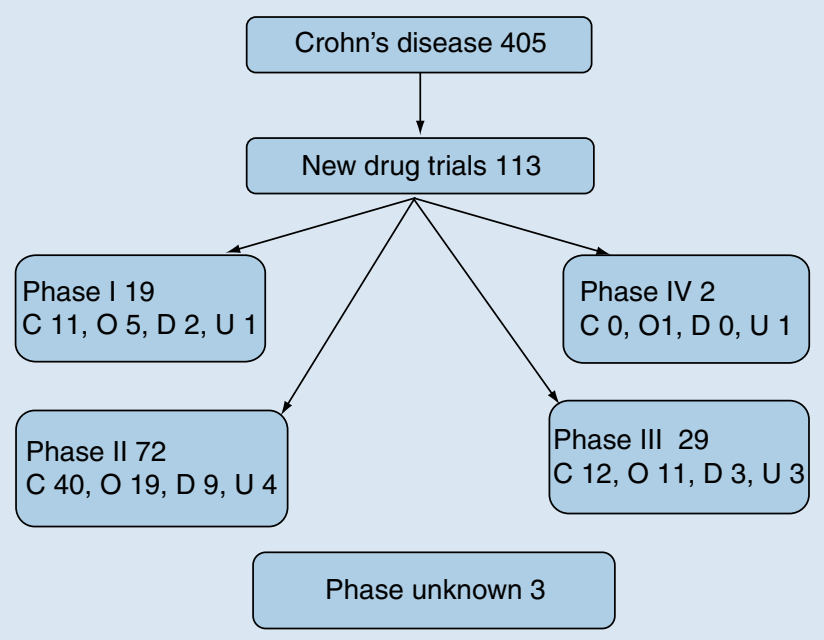

Figure 1. Discontinued trials in Crohn's disease. All trials on ClinicalTrials.gov listed under Crohn's disease. Of these trials, 113 trials were on new drugs. New drug trials were subdivided according to trial phase, and further divided into completed trials (C ), ongoing trial (O), discontinued trials $(D)$ and trial status unknown $(U)$. for finding full publications regarding the indentified trials with the following search criteria: ..(the drug).. AND IBD, or ..(the drug).. AND inflammatory bowel disease, or ..(the drug).. AND Crohn's disease, or ..(the drug)..AND ulcerative colitis, or ..(the drug) AND colitis. The presented abstracts of the DDW 2011 congress and the ECCO 2011 congress were searched for unpublished data on the identified drugs.

\section{Results}

For CD 405 clinical trials were identified, of these 113 investigated new drugs. For nine trials no follow-up results were available and were thus excluded (Figure 1). For UC 282 clinical trials were identified, of which 78 concerned researching new drugs. Due to absence of follow-up results five trials were excluded (Figure 2). Trials with an unknown phase were excluded as well. Combination trials (i.e., Phase I/II and Phase II/III trials) were included.

\section{Overview of discontinued trials}

Thirty-four Phase I trials were identified (Figure 3), 13 were combined Phase I/II trials. Four trials (two CD/ two UC/two combined Phase I/II) were discontinued and included for further analysis. The drugs researched in these Phase I trials were sargramostim, givinostat, RhIL-11 and GI-270384.

In Phase II, 123 trials were commenced according to clinicaltrials.gov. Of these, 13 were Phase I/II trials and another 13 were Phase II/III, the remaining were true Phase II trials. A total of 18 trials (nine CD/ nine UC) were discontinued. Two were Phase I/II and three were Phase II/III. Researched drugs were sargramostim, tetomilast, AIN-457, semapinod, brakinumab, ITF-2357, SB-656933, visilizumab, basiliximab, riverprost, golimumab and retard release phosphatidylcholine and IFN- $\beta-1 \alpha$.

For Phase III trial, 46 trials were identified (Supplementary Table 1). In total, 13 were combined Phase II/III trials. A total of seven trials were discontinued. Of these trials, three were CD, four were UC and four trials were Phase II/III trials. Researched drugs were sagramostim, abatacept, visilizumab, golimumab and retard release phosphatidylcholine.

In total, 24 discontinued trials on new drugs were included, of which two were Phase I/II and three were Phase II/III. The 24 trials were on 16 different new drugs. Lack of efficacy was the reason for trial discontinuation in 10 out of 16 treatments (62.5\%).

\section{Discussion}

In total, 191 trials on novel drugs were mentioned on the Clinicaltrials.gov website [101], 24 (12.6\%) were interrupted. The most common reason for discontinuation 
was lack of efficacy (Supplementary Table 1). Further analysis of these discontinued trials led to questions regarding the current trial design, such as; how to translate results from animal studies to patient populations, and, is it feasible to start treatment with drugs while still unaware of the full safety profile?

New treatments are designed in laboratories and translated to humans based on a positive effect found in cell lines and animal models. However, these positive results cannot be readily translated to the patient, and can even lead to deleterious effects. For example, murine anti-CD3 antibody, OKT 3, showed side-effects associated with severe systemic cytokine release [15]. In addition, although visilizumab has been altered to specifically bind to human CD3 expressed on T-cells leaving the quiescent T-cells unchanged in vitro, a Phase I study of visilizumab demonstrated at least one adverse event in the majority of patients, with a quarter experiencing severe adverse events $[16,17]$. Another challenge is the effectiveness of drugs: IL-11 decreased histological as well as clinical activity of colitis in animals, but in humans this drug was only effective in a subset of CD patients [18,19], which shows that results of animal studies can also be applicable only for a group of our patients. Issues considering effectiveness were also seen investigating tetomilast. Although effective on the bench, UC patients did not improve compared with the placebo-treated patients [20,21]. Similar are the results of trials in sargramostim. This drug was found to be effective in mice [22], but the same effect could not be confirmed in humans [23,24]. Another problem faced, is that various models are used for investigating drugs, leading to difficulties in comparing outcomes. This is illustrated by the fact that IL-17a knockout mice and IL-17RA knockout mice showed less inflammation after treatment with a neutralizing anti-IL-17A antibody $[25,26]$, but antiIL-17a antibodies showed worsening of disease in mice with dextran sulfate sodium colitis [27]. In line with this are the results of rivenprost. In animals, both an EP4R agonist and an antagonist turned out to have an immunosuppressive effect [28,29]. A trial in humans was started before results of the antagonist were known and rivenprost was found to be ineffective in humans. Overall these results demonstrate that models for diseases come with restrictions and the pathogenesis of inflammation in these models does not adequately resemble IBD patients.

\section{Future perspective}

An even closer collaboration between workers on the bench and workers at the bedside is advocated to overcome problems such as those described above.

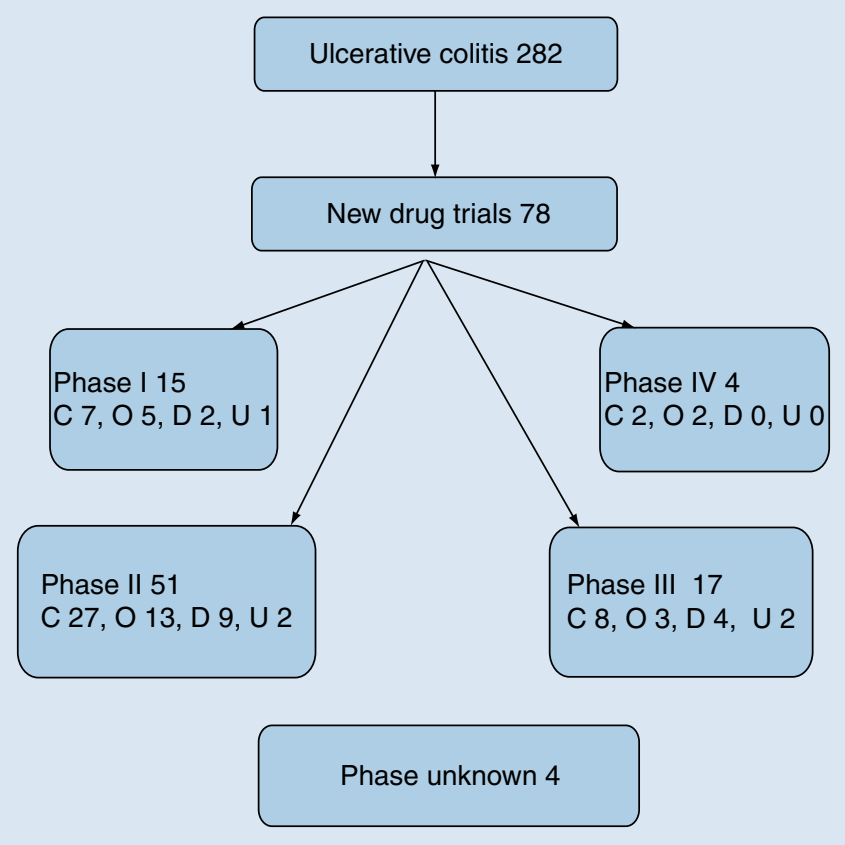

Figure 2. Discontinued trials in ulcerative colitis. All trials on ClinicalTrials.gov listed under ulcerative colitis. Of these trials, 78 trials were on new drugs. New drug trials were subdivided according to trial phase, and further divided into completed trials (C), ongoing trial (O), discontinued trials (D) and trial status unknown (U).

However, future trial design also needs to be redirected to implement other earlier published results on trial design. Poynard et al. looked at the survival of truth of clinical conclusions in articles on cirrhosis and hepatitis and found the half-life to be 45 years, similar to an earlier published report [30,31]. The authors did not find a beneficial survival benefit for either randomized trials or meta-analysis. The biggest weight (four-times more than other trials) was given to negative trials where patients with severe disease were mixed with patients without severe disease. In order to provide a trial with true power, future trial cohorts should resemble the patient population, including their genetic heterogeneity [32-35]. This heterogeneity may explain that the effect of new drugs can differ in each individual patient. Recently we showed that granulocytes of CD patients with a specific genetic defect are less sensitive to stimulation with GM-CSF. Therefore a small proportion of patients without this defect may actually benefit from GM-CSF treatment [36]. Also trials on IFN- $\beta-1 \alpha$ show similar results. At first, IFN- $\beta-1 \alpha$ did not seem to be effective in either CD nor UC [37,38]. However, after looking at the results more closely, Croatian and Russian patients did have high remission and response rates using this treatment, while western 


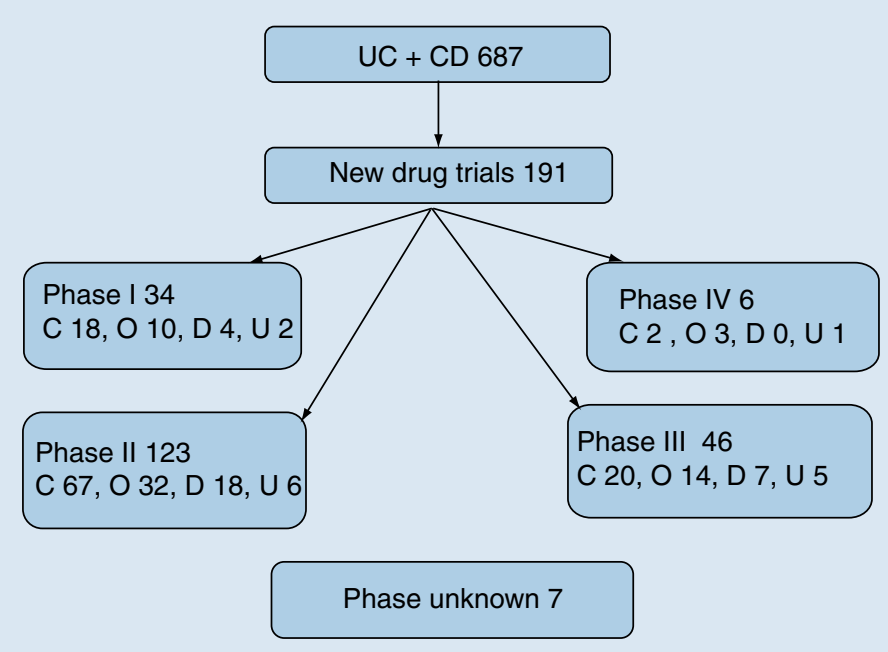

Figure 3. Total discontinued trials in inflammatory bowel disease. Total number of trials on ClinicalTrials.gov, listed under either CD or UC and the total number of trials on new drugs. The subdivision was the same as shown in the previous figures. is unknown. Currently the placebo effect in $\mathrm{CD}$ and UC is well known and can be influenced by adjusting the study design, for instance [40-45]. Today, the standard design for developing new therapies are studies based on superiority tests in which a new therapy is compared with a control or current standard of care in a randomized setting with the aim of improving efficacy. Future design should incorporate comparisons of new therapy, not only with placebo, but also to the current therapy standard in a three-arm design [46].

Currently, there is a suboptimal climate for publication when it comes to new drugs. Only a quarter of studies on new drugs are published at the time of market authorization and the majority of those are studies with positive findings [47]. The pharmaceutical industry also plays a large role in designing, conducting and discontinuation of trials on new drugs. The industry would rather see trials on promising drugs published, since negative results might negatively affect drug sales. This is understandable from their point of view. However, in order to provide the best possible patient care, the negative results are also worth mentioning, as has been proved earlier in this paper. To resolve this problem, someone, either a regulator, or an independent board, should be able to require submission of full trial data. This person or board should also be able to enforce sharing all available data [48]. This policy will not only improve the quality of newly conducted drug trials, but will also facilitate the comparison of new and existing drugs.

\section{Conclusion}

Of the 16 drugs investigated in the discontinued trials, ten did lack efficacy in IBD patients, despite

Executive summary

\section{Background}

- Anti-TNF- $\alpha$ drugs are very potent for the majority of our inflammatory bowel disease patients but the current therapeutic arsenal still does not fully fulfill the therapeutic needs of our patients. However, many drugs will never reach the market. We elucidate pitfalls in clinical trials and offer recommendation for future research.

\section{Materials \& methods}

- On ClinicalTrials.gov interventional trials on novel therapies that could be of interest for the treatment of inflammatory bowel disease were reviewed. Included were discontinued trials mentioned under the conditions listed under the terms 'Crohn's disease' and/or 'colitis, ulcerative'. The trials were started between July 1996 and October 2011. After inclusion of the most potent trials Pubmed was searched for full publication of these trials. Abstracts presented on the DDW 2011 congress and the ECCO 2011 congress were searched for unpublished data on the found drugs.

Results

- 191 novel drug trials were published on ClinicalTrials.gov, of which 24 (12.6\%) were discontinued. The most common reason for discontinuation was lack of efficacy.

\section{Conclusion}

- Translating from bench to bedside is not always feasible. Animal models come with restrictions. In the future, personalized medicine based on a patient genetic profile will become important. Knowledge about interrupted trials is necessary to conduct high quality new drug trials in the future. 
the promising results in models. This shows the difficulties in translating results from bench to bedside. Therefore, stronger collaboration between bench and bedside workers is required.

In the future, the cohorts must be a resemblance of the patient population, since these trials have a longer truth survival. Furthermore, genetics will start playing a bigger role and the role for the placebo-compared testing will become less significant. Personalized medicine based on a patient genetic profile and biologically important pathways are the future in IBD. Truth survival comes with change in trial methodology. Knowledge about interrupted trials is necessary to conduct high quality new drug trials in the future.

\begin{abstract}
Supplementary data
Supplementary data accompanies this paper and can be found at www.future-science.com/doi/suppl/10.4155/ CLI.11.185

\section{Financial \& competing interests disclosure \\ VJAA Nuij has received Dr Falk Benelux bv. unrestricted educational grant. CJ van der Woude has received Abbott Laboratories consulting fees and worked on their Advisory Committees or Review Panels; Schering-Plough Corp./MSD consulting fees and worked on their Advisory Committees or Review Panels; Shire Pharmaceuticals Inc., consulting fees and worked on their Advisory Committees or Review Panels; and a Dr Falk Benelux bv. grant. The authors have no other rele- vant affiliations or financial involvement with any}

organization or entity with a financial interest in or financial conflict with the subject matter or materials discussed in the manuscript apart from those disclosed.

No writing assistance was utilized in the production of this manuscript.

\section{References}

Papers of special note have been highlighted as:

- of interest

. of considerable interest

1 Langholz E, Munkholm P, Davidsen M, Nielsen OH, Binder V. Changes in extent of ulcerative colitis: a study on the course and prognostic factors. Scand. J. Gastroenterol. 31(3), 260-266 (1996).

2 Langholz E, Munkholm P, Davidsen M, Binder V. Course of ulcerative colitis: analysis of changes in disease activity over years. Gastroenterology 107(1), 3-11 (1994).

3 Cosnes J, Cattan S, Blain A et al. Long-term evolution of disease behavior of Crohn's disease. Inflamm. Bowel Dis. 8(4), 244-250 (2002).

4 Loftus EV Jr, Schoenfeld P, Sandborn WJ. The epidemiology and natural history of Crohn's disease in population-based patient cohorts from North America: a systematic review. Aliment Pharmacol. Ther. 16(1), 51-60 (2002).

5 Loftus EV Jr. Clinical epidemiology of inflammatory bowel disease: incidence, prevalence and environmental influences. Gastroenterology 126(6), 1504-1517 (2004).

6 Koelewijn C, Schrijvers A, Oldenburg B. Infliximab use in patients with Crohn's disease: quality of life, costs and resource use. Neth. J. Med. 64(7), 212-218 (2006).

7 Caviglia R, Ribolsi M, Rizzi M, Emerenziani
S, Annunziata ML, Cicala M. Maintenance of remission with infliximab in inflammatory bowel disease: efficacy and safety long-term follow-up. World J. Gastroenterol. 13(39), 5238-5244 (2007).

8 Rutgeerts P, Van Assche G, Vermeire S. Review article: infliximab therapy for inflammatory bowel disease - seven years on. Aliment Pharmacol. Ther. 23(4), 451-463 (2006).

9 Gonzalez-Lama Y, Lopez-San Roman A, Marin-Jimenez I et al. Open-label infliximab therapy in Crohn's disease: a long-term multicenter study of efficacy, safety and predictors of response. Gastroenterol. Hepatol. 31(7), 421-426 (2008).

10 Cosnes J, Nion-Larmurier I, Beaugerie L, Afchain P, Tiret E, Gendre JP. Impact of the increasing use of immunosuppressants in Crohn's disease on the need for intestinal surgery. Gut 54(2), 237-241 (2005).

11 Hanauer SB, Feagan BG, Lichtenstein GR et al. Maintenance infliximab for Crohn's disease: the ACCENT I randomised trial. Lancet 359(9317), 1541-1549 (2002).

12 Farrell RJ, Alsahli M, Jeen YT, Falchuk KR, Peppercorn MA, Michetti P. Intravenous hydrocortisone premedication reduces antibodies to infliximab in Crohn's disease: a randomized controlled trial. Gastroenterology 124(4), 917-924 (2003).

13 Colombel JF, Loftus EV Jr, Tremaine WJ et al. The safety profile of infliximab in patients with Crohn's disease: the Mayo clinic experience in 500 patients. Gastroenterology 126(1), 19-31 (2004).

14 Hanauer SB, Sandborn WJ, Rutgeerts P et al. Human anti-tumor necrosis factor monoclonal antibody (adalimumab) in Crohn's disease: the CLASSIC-I trial.
Gastroenterology 130(2), 323-333; quiz 591 (2006).

15 Cosimi AB, Burton RC, Colvin RB et al. Treatment of acute renal allograft rejection with OKT3 monoclonal antibody. Transplantation 32(6), 535-539 (1981).

16 Cole MS, Stellrecht KE, Shi JD et al. HuM291, a humanized anti-CD3 antibody, is immunosuppressive to $\mathrm{T}$ cells while exhibiting reduced mitogenicity in vitro. Transplantation 68(4), 563-571 (1999).

17 Plevy S, Salzberg B, Van Assche G et al. A Phase I study of visilizumab, a humanized anti-CD3 monoclonal antibody, in severe steroid-refractory ulcerative colitis. Gastroenterology 133(5), 1414-1422 (2007).

18 Peterson RL, Wang L, Albert L et al. Pharmacogenomic analysis of rhIL-11 treatment in the HLA-B27 rat model of inflammatory bowel disease. Pharmacogenomics J. 2(6), 383-399 (2002).

19 Sands BE, Winston BD, Salzberg B et al. Randomized, controlled trial of recombinant human interleukin-11 in patients with active Crohn's disease. Aliment Pharmacol. Ther. 16(3), 399-406 (2002).

20 Ichikawa $\mathrm{H}$, Okamoto S, Kamada $\mathrm{N}$ et al. Tetomilast suppressed production of proinflammatory cytokines from human monocytes and ameliorated chronic colitis in IL-10-deficient mice. Inflamm. Bowel Dis. 14(11), 1483-1490 (2008).

21 Schreiber S, Keshavarzian A, Isaacs KL et al. A randomized, placebo-controlled, Phase II study of tetomilast in active ulcerative colitis. Gastroenterology 132(1), 76-86 (2007).

22 Sainathan SK, Hanna EM, Gong Q et al. Granulocyte macrophage colonystimulating factor ameliorates DSS-induced 
experimental colitis. Inflamm. Bowel Dis. 14(1), 88-99 (2008).

23 Korzenik JR, Dieckgraefe BK, Valentine JF, Hausman DF, Gilbert MJ. Sargramostim for active Crohn's disease. N. Engl. J. Med. 352(21), 2193-2201 (2005).

24 Takazoe M, Matsui T, Motoya S, Matsumoto T, Hibi T, Watanabe M. Sargramostim in patients with Crohn's disease: results of a Phase I-II study. J. Gastroenterol. 44(6), 535-543 (2009).

25 Ito R, Kita M, Shin-Ya M et al. Involvement of IL-17A in the pathogenesis of DSSinduced colitis in mice. Biochem. Biophys. Res. Commun. 377(1), 12-16 (2008).

- Demonstrates that care should be taken when translating from bench to bedside.

26 Zhang Z, Zheng M, Bindas J, Schwarzenberger P, Kolls JK. Critical role of IL-17 receptor signaling in acute TNBSinduced colitis. Inflamm. Bowel Dis. 12(5), 382-388 (2006).

- Demonstrates that care should be taken when translating from bench to bedside.

27 Ogawa A, Andoh A, Araki Y, Bamba T, Fujiyama Y. Neutralization of interleukin-17 aggravates dextran sulfate sodium-induced colitis in mice. Clin. Immunol. 110(1), 55-62 (2004).

- Demonstrates that care should be taken when translating from bench to bedside.

28 Yao C, Sakata D, Esaki Y et al. Prostaglandin E2-EP4 signaling promotes immune inflammation through Th1 cell differentiation and Th17 cell expansion. Nat. Med. 15(6), 633-640 (2009).

29 Jiang GL, Nieves A, Im WB, Old DW, Dinh DT, Wheeler L. The prevention of colitis by E Prostanoid receptor 4 agonist through enhancement of epithelium survival and regeneration. J. Pharmacol. Exp. Ther. 320(1), 22-28 (2007).

30 Poynard T, Munteanu M, Ratziu V et al. Truth survival in clinical research: an evidence-based requiem? Ann. Intern. Med. 136(12), 888-895 (2002).

-. A very nice article on the value of clinical research.
31 Hall JC, Platell C. Half-life of truth in surgical literature. Lancet 350(9093), 1752 (1997).

32 Hampe J, Franke A, Rosenstiel P et al. A genome-wide association scan of nonsynonymous SNPs identifies a susceptibility variant for Crohn disease in ATG16L1. Nat. Genet. 39(2), 207-211 (2007).

33 Parkes M, Satsangi J, Lathrop GM, Bell JI, Jewell DP. Susceptibility loci in inflammatory bowel disease. Lancet 348(9041), 1588 (1996).

34 Gaya DR, Russell RK, Nimmo ER, Satsangi J. New genes in inflammatory bowel disease: lessons for complex diseases? Lancet 367(9518), 1271-1284 (2006).

35 Barrett JC, Hansoul S, Nicolae DL et al. Genome-wide association defines more than 30 distinct susceptibility loci for Crohn's disease. Nat. Genet. 40(8), 955-962 (2008).

36 Somasundaram R, Deuring JJ, Van Der Woude CJ, Peppelenbosch MP, Fuhler GM. Linking risk conferring mutations in NCF4 to functional consequences in Crohn's disease. Gut doi:10.1136/gutjnl-2011-301344 (2011) (Epub ahead of print).

- A nice letter about the functional consequences of a mutation in a risk allele.

37 Pena Rossi C, Hanauer SB, Tomasevic R, Hunter JO, Shafran I, Graffner H. Interferon beta-1a for the maintenance of remission in patients with Crohn's disease: results of a Phase II dose-finding study. $B M C$ Gastroenterol. 9, 22 (2009).

- When analyzing the results in this article more closely, there might be a benefit for a specific patient population.

38 Pena-Rossi C, Schreiber S, Golubovic G et al. Clinical trial: a multicentre, randomized, double-blind, placebo-controlled, dosefinding, Phase II study of subcutaneous interferon-beta-la in moderately active ulcerative colitis. Aliment Pharmacol. Ther. 28(6), 758-767 (2008).

- When analyzing the results in this article more closely, there might be a benefit for a specific patient population.

39 Mannon PJ, Hornung RL, Yang Z et al.
Suppression of inflammation in ulcerative colitis by interferon-beta-1a is accompanied by inhibition of IL-13 production. Gut 60(4), 449-455 (2011).

40 Su C, Lewis JD, Goldberg B, Brensinger C, Lichtenstein GR. A meta-analysis of the placebo rates of remission and response in clinical trials of active ulcerative colitis. Gastroenterology 132(2), 516-526 (2007).

$41 \mathrm{Su}$ C. Outcomes of placebo therapy in inflammatory bowel disease. Inflamm. Bowel Dis. 12(4), 328-333 (2006).

42 Meyers S, Janowitz HD. 'Natural history' of Crohn's disease. An analytic review of the placebo lesson. Gastroenterology 87(5), 1189-1192 (1984).

43 Sands BE. The placebo response rate in irritable bowel syndrome and inflammatory bowel disease. Dig. Dis. 27(Suppl. 1), 68-75 (2009).

44 Su C, Lichtenstein GR, Krok K, Brensinger CM, Lewis JD. A meta-analysis of the placebo rates of remission and response in clinical trials of active Crohn's disease. Gastroenterology 126(5), 1257-1269 (2004).

45 Meyers S, Janowitz Hd. The 'natural history' of ulcerative colitis: an analysis of the placebo response. J. Clin. Gastroenterol. 11(1), 33-37 (1989).

46 Ulrich Burger H, Beyer U, Abt M. Issues in the assessment of non-inferiority: perspectives drawn from case studies. Pharm. Stat. 10(5), 433-439 (2011).

47 Van Luijn JC, Stolk P, Gribnau FW, Leufkens HG. Gap in publication of comparative information on new medicines. Br. J. Clin. Pharmacol. 65(5), 716-722 (2008).

- Nicely ellucidates the problem of comparing new medicines.

48 Garattini S, Bertele V. Europe's opportunity to open up drug regulation. $B M J$ 340, c1578 (2010).

- Provides possible solutions to the problems mentioned in reference 47.

\section{- Website}

101 ClinicalTrials.gov www.clinicaltrials.gov 